\title{
Compromised genomic integrity impedes muscle growth after Atrx inactivation
}

\author{
Michael S. Huh, ${ }^{1}$ Tina Price O’Dea, ${ }^{1}$ Dahmane Ouazia, ${ }^{1}$ Bruce C. McKay, ${ }^{2,3}$ Gianni Parise, ${ }^{4}$
} Robin J. Parks, ${ }^{1,3}$ Michael A. Rudnicki,, ${ }^{1,3}$ and David J. Picketts ${ }^{1,3}$

\begin{abstract}
${ }^{1}$ Regenerative Medicine Program and ${ }^{2}$ Cancer Therapeutics Program, Ottawa Hospital Research Institute, Ottawa, Ontario, Canada. ${ }^{3}$ Department of Medicine, University of Ottawa, Ottawa, Ontario, Canada. ${ }^{4}$ Department of Kinesiology and Department of Medical Physics and Applied Radiation Sciences,
\end{abstract} McMaster University, Hamilton, Ontario, Canada.

\begin{abstract}
ATR-X syndrome is a severe intellectual disability disorder caused by mutations in the ATRX gene. Many ancillary clinical features are attributed to CNS deficiencies, yet most patients have muscle hypotonia, delayed ambulation, or kyphosis, pointing to an underlying skeletal muscle defect. Here, we identified a cell-intrinsic requirement for Atrx in postnatal muscle growth and regeneration in mice. Mice with skeletal muscle-specific Atrx conditional knockout (Atrx cKO mice) were viable, but by 3 weeks of age presented hallmarks of underdeveloped musculature, including kyphosis, $20 \%$ reduction in body mass, and $34 \%$ reduction in muscle fiber caliber. Atrx cKO mice also demonstrated a marked regeneration deficit that was not due to fewer resident satellite cells or their inability to terminally differentiate. However, activation of Atrx-null satellite cells from isolated muscle fibers resulted in a 9-fold reduction in myoblast expansion, caused by delayed progression through mid to late $S$ phase. While in $S$ phase, Atrx colocalized specifically to late-replicating chromatin, and its loss resulted in rampant signs of genomic instability. These observations support a model in which Atrx maintains chromatin integrity during the rapid developmental growth of a tissue.
\end{abstract}

\section{Introduction}

Males inheriting germline mutations in the ATRX gene develop $\alpha$-thalassemia mental retardation X-linked (ATR-X) syndrome (1). ATR-X syndrome is a human developmental disorder characterized by severe intellectual disabilities, $\alpha$-thalassemia, urogenital dysfunction, skeletal abnormalities, and neonatal hypotonia. This characteristic collection of symptoms in patients suggests a critical role for ATRX in these tissues. Similarly, in mice, the survival of neurons in the CNS and the development of reproductive tissue also requires Atrx (2-5).

The ATRX gene encodes a $280-\mathrm{kDa}$ chromatin remodeling protein with an N-terminal ATRX-DNMT3-DNMT3L (ADD) domain that forms a histone binding pocket and a C-terminal SNF2 ATPase domain (6-9). Like most SNF2 chromatin remodelers, ATRX is part of a larger complex that includes the death domainassociated protein (Daxx) $(10,11)$. Chromatin remodeling complexes in general use the energy derived from ATP hydrolysis to reorganize nucleosome position, promote disassembly/incorporation of nucleosomes during DNA replication, and actively facilitate histone variant exchange $(12,13)$. Histone variants are incorporated into nucleosomes throughout the cell cycle, unlike the replication-dependent canonical histones. Structural incorporation of histone variants often accompanies a functional change in chromatin. For example, deposition of histone variant macroH2A is concomitant with facultative silencing of the female $\mathrm{X}$ chromosome (14). In contrast, histone variant H3.3 is highly enriched at transcribed genes and in the constitutive heterochromatin found at pericentromeres and telomeres (15-19). Atrx-Daxx complexes are required for the deposition of histone variant $\mathrm{H} 3.3$ at pericentromeres and telomeres, but, strangely, not in transcribed genes (17). Furthermore, Atrx ChIP sequencing experiments by Law et al.

Conflict of interest: The authors have declared that no conflict of interest exists. Citation for this article: J Clin Invest. 2012;122(12):4412-4423. doi:10.1172/JCI63765. demonstrated an affinity for G-rich and simple tandem repeats (TRs) found in telomeres, $\alpha$-globin locus, and throughout the genome (20). Genome-wide occupancy at TRs by Atrx suggests a global role in regulating chromatin structure and genome integrity. Intriguingly, somatic mutations in Atrx have been found in acquired $\alpha$-thalassemia myelodysplastic syndrome (ATMDS) and, more recently, in pancreatic neuroendocrine tumors (PanNETs), where $61 \%$ of PanNETs examined exhibited abnormal telomeres reminiscent of tumors that activate the alternative lengthening of telomeres (ALT) pathway (21-24).

Here, we explored the role of Atrx in skeletal muscle development, as neonatal hypotonia is diagnosed in $85 \%$ of all ATR-X syndrome patients (25). Due to their reduced muscle function, patients do not walk until later in childhood, while some remain incapable for a lifetime. We showed a primary defect in muscle growth and regeneration caused by an accumulation of genomic damage in Atrx-deficient satellite cells. Despite normal resting numbers, Atrx-knockout satellite cells were incapable of sustained expansion in ex vivo muscle fiber cultures. 2-parameter flow cytometry BrdU pulse-chase analysis of Atrx-knockout satellite cell-derived myoblasts revealed a delay in the progression through mid to late $S$ phase, implicating a replication-dependent chromatin defect. Consequently, we observed fragmented and aberrant nuclei, elevated levels of $\gamma$-H2AX foci, telomeric fragility, and activation of the p53-ataxia-telangiectasia mutated (p53-ATM) DNA damage response (DDR). Taken together, these observations indicate that Atrx is required for replication-dependent chromatin maintenance in rapidly proliferating myoblasts.

\section{Results}

Atrx conditional knockout mice have delayed muscle growth and severe deficits in muscle regeneration. Skeletal muscle hypotonia, delayed ambulation, and kyphosis are commonly associated with ATR-X syndrome patients (1). Therefore, we postulated that Atrx func- 

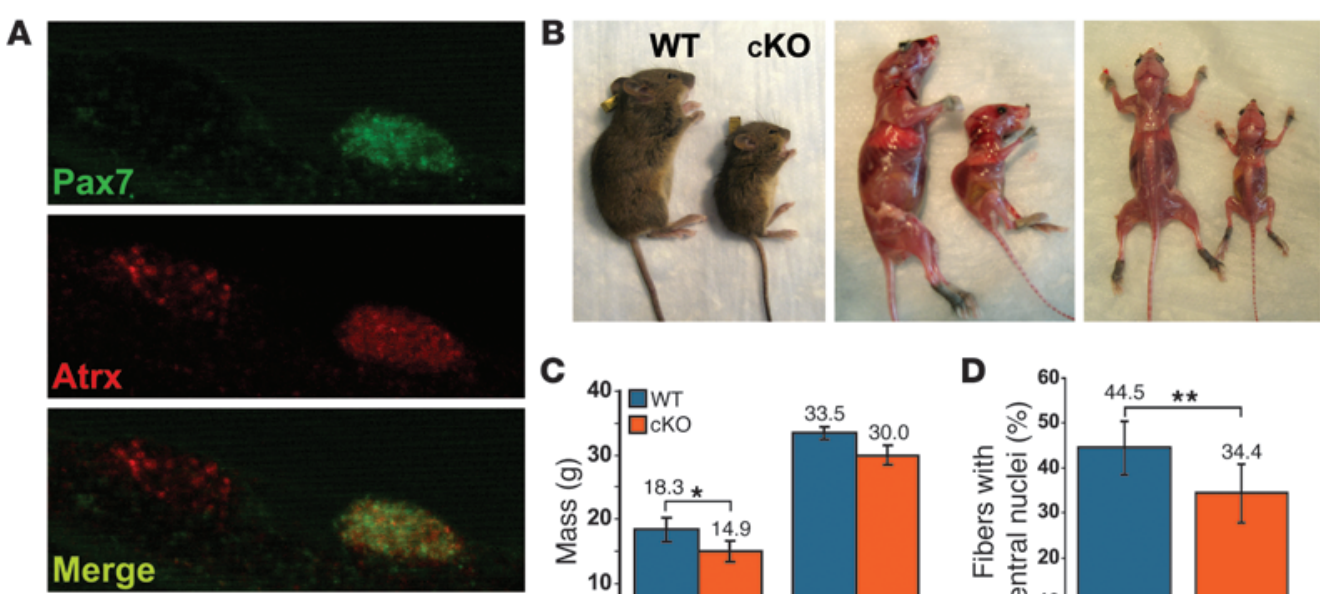

c
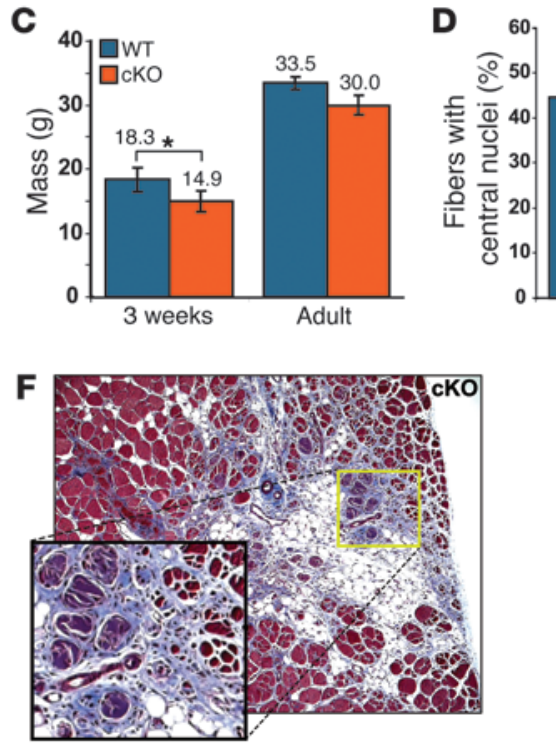

Figure 1

Atrx inactivation in skeletal muscle leads to delay in muscle growth and severe regeneration deficit after CTXinduced acute injury. (A) Confocal double-immunofluorescent micrograph of Atrx and Pax7 from a freshly isolated EDL myofiber. (B) 3-week-old Atrx CKO and Atrx f/y control (WT) mice. (C) Body mass comparison between Atr $X^{f / y}$ and Atrx CKO mice at 3 weeks and 8 weeks (Adult) of age. Values represent mean $\pm \operatorname{SEM}(n=9)$. ${ }^{*} P<0.05, t$ test. (D) Quantification of regenerating muscle fibers as assessed by the proportion of nuclei located centrally in muscle fibers 2 weeks after CTX injection. ${ }^{* \star} P<0.01, t$ test. (E and F) Masson's Trichrome staining of the TA muscle 2 weeks after CTX injection from Atrx ${ }^{f / y}(\mathbf{E})$ and Atrx CKO (F) mice. Poor muscle regeneration in Atrx cKO mice is shown by the excessive amounts of fibrotic tissue (blue collagen staining) in the damaged region (F, inset). Original magnification, $\times 630(\mathbf{A}) ; \times 100(\mathbf{E}$ and $\mathbf{F})$. Insets in $\mathbf{E}$ and $\mathbf{F}$ are enlarged $\times 3$.

deviation from the expected Mendelian ratios for all the predicted genotypes (Supplemental Table 1; supplemental material available online with this article; doi:10.1172/ JCI63765DS1). Since Atrx cKO mice were viable, it appeared that embryonic and fetal myogenesis was generally unaffected in our model.

Nonetheless, Atrx cKO mice were consistently smaller in size than their Atr $x^{f} / y$ littermate controls from birth (Figure 1B). Analysis at 3 weeks of age demonstrated a $20 \%$ reduction in body mass, which correlated with an overall reduction in limb musculature and exaggerated curvature of the spine (Figure 1, B and C). These observations indicate that Atrx is important for the growth and maturation of skeletal muscle in young mice. Interestingly, by adulthood, differences in body mass became insignificant between Atrx cKO mice and Atr $x^{f / y}$ littermate controls (Figure 1C).

Postnatal growth delay may indicate that satellite cell number or function is compromised. Satellite cells are also responsible for the robust regenerative capacity of adult skeletal muscle. We therefore assessed the recovery of adult Atrx cKO muscle from an injury. Acute injury was

tion would be essential for the normal growth and development of skeletal muscle. We thus sought to determine whether Atrx is expressed in vivo in skeletal muscle fibers. Coimmunofluorescent staining for Atrx and the satellite cell marker Pax7 revealed that Atrx was expressed in both quiescent satellite cells and differentiated myonuclei (Figure 1A). Interestingly, expression of Atrx appeared to be more intense in satellite cell nuclei than in differentiated myonuclei. Moreover, the Atrx nuclear staining pattern was largely homogenous, with distinct foci in satellite cells, contrasting with a strictly punctate focal pattern in differentiated myonuclei.

To further explore a role for Atrx, we generated skeletal muscle-specific conditional knockout of Atrx by interbreeding mice harboring a Cre recombinase knockin within the locus of the myogenic regulatory factor $M y f 5$ (26). $M y f 5^{\mathrm{Cre} /+}$ males were crossed with homozygously floxed Atrx females (Atrxf/f; ref. 27). X-linkage of Atrx ensured that all male progeny carried the Atrx floxed allele $\left(A T R X^{f} / y\right)$, of which half were expected to be $\mathrm{Cre}^{+}$, and thus genetically designated Atrxf/y:Myf5-Cre (referred to herein as Atrx cKO). The progeny generated from this cross resulted in no significant induced in the tibialis anterior (TA) muscle by cardiotoxin (CTX) injection in Atrx cKO and Atrxf/y littermate controls. Recovery from CTX injury was clearly abnormal in Atrx cKO mice. Atrx cKO TA muscles displayed an abundance of poorly formed microfibers and open interstitial cavities in the area of regeneration 2 weeks after CTX injury, in contrast to the fully regenerated fibers from Atrxf/y littermates (Figure 1, E and F). A significantly lower proportion of muscle fibers contained centrally located nuclei 2 weeks after CTX (Figure 1D), indicative of a reduction of regenerating muscle fibers. Furthermore, fiber caliber measurements before and after CTX demonstrated an inability of Atrx cKO mice to efficiently increase both the overall proportion (Atrx cKO, 9\% increase; Atr $x^{f / y}, 22 \%$ increase) and size (Atrx cKO, 25\% increase; Atr $x f / y, 83 \%$ increase) of muscle fibers with cross-sectional area greater than $0.1 \mathrm{~mm}^{2}$ (Table 1). The presence of highly fibrotic regeneration was also strikingly evident by Masson's Trichrome staining in Atrx cKO, but not Atr $x^{f / y}$, TA muscles (Figure 1, E and F). The early postnatal growth delay and poor muscle regeneration after CTX injury point to possible defects in satellite cell number or function in Atrx cKO mice. 


\section{Table 1}

Fiber caliber measurements of uninjured and CTX-injured TA muscle in adult AtrxcKO mice and Atr $x^{f / y}$ littermate controls

\begin{tabular}{lcccc}
\hline & \multicolumn{2}{c}{ Small $\left(<\mathbf{0 . 1} \mathbf{~ m m}^{2}\right)$} & \multicolumn{2}{c}{ Large $\left(>\mathbf{0 . 1} \mathbf{~ m m}^{2}\right)$} \\
& Area $\left(\mathbf{m m}^{2}\right)$ & Frequency & Area $\left(\mathbf{m m}^{2}\right)$ & Frequency \\
Atrx ${ }^{f y}$ uninjured & $0.054 \pm 0.02$ & $67 \%$ & $0.120 \pm 0.02$ & $33 \%$ \\
Atrx ${ }^{f y}$ CTX & $0.054 \pm 0.03$ & $45 \%$ & $0.219 \pm 0.09$ & $55 \%$ \\
Atrx CKO uninjured & $0.062 \pm 0.02$ & $79 \%$ & $0.114 \pm 0.02$ & $21 \%$ \\
Atrx cKO CTX & $0.045 \pm 0.02$ & $70 \%$ & $0.142 \pm 0.03$ & $30 \%$ \\
\hline
\end{tabular}

Area values are mean \pm SEM

Atrx cKO muscle has normal numbers of satellite cells, but compromised growth properties. Satellite cells represent the primary source of muscle progenitors for postnatal muscle growth and regeneration (28). We therefore set out to determine the presence and functionality of the satellite cells in our Atrx cKO mouse model. Myf5-Credriven targeted excision was assessed in a Cre-activated ROSA-YFP reporter mouse (Myf5-Cre:ROSA-YFP). Staining of freshly isolated muscle fibers from Myf5-Cre:ROSA-YFP mice revealed coexpression of Atrx in $\mathrm{YFP}^{+}$satellite cells (Supplemental Figure 1A).
Atrx immunostaining on freshly isolated Atrx cKO fibers verified complete Cre excision in the skeletal muscle lineage (Figure 2A). Despite the absence of Atrx, the percentage of $\mathrm{Pax} 7^{+}$satellite cells was not significantly different in bulk hind limb muscle digests from 2-week-old Atrx cKO mice and Atr $f^{f / y}$ littermate controls (Supplemental Figure 1B). Furthermore, direct counts of Pax $7^{+}$nuclei from freshly isolated extensor digitorum longus (EDL) muscle fibers confirmed the presence of normal numbers of satellite cells in both Atrx cKO and Atrx f/y animals (Figure 2B). Our satellite cell counts were consistent with the numbers reported in other studies, which range between 8 and 10 satellite cells per EDL fiber $(29,30)$.

Since the absolute numbers of resident satellite cells did not appear to vary significantly in Atrx cKO mice, we proceeded to characterize the growth properties of activated satellite cells from live single myofiber cultures. Myofibers were maintained in a nonadherent floating culture system supplemented with BrdU. Satellite cell activation and subsequent myoblast division were marked by BrdU incorporation. Each individual fiber represented a single unit giving rise to an isolated population of daughter myoblasts. After 48 hours in culture, Atrx cKO fibers generated a modest number of $\mathrm{BrdU}^{+}$myoblasts, albeit significantly lower than that of Atr $x^{f / y}$ con-
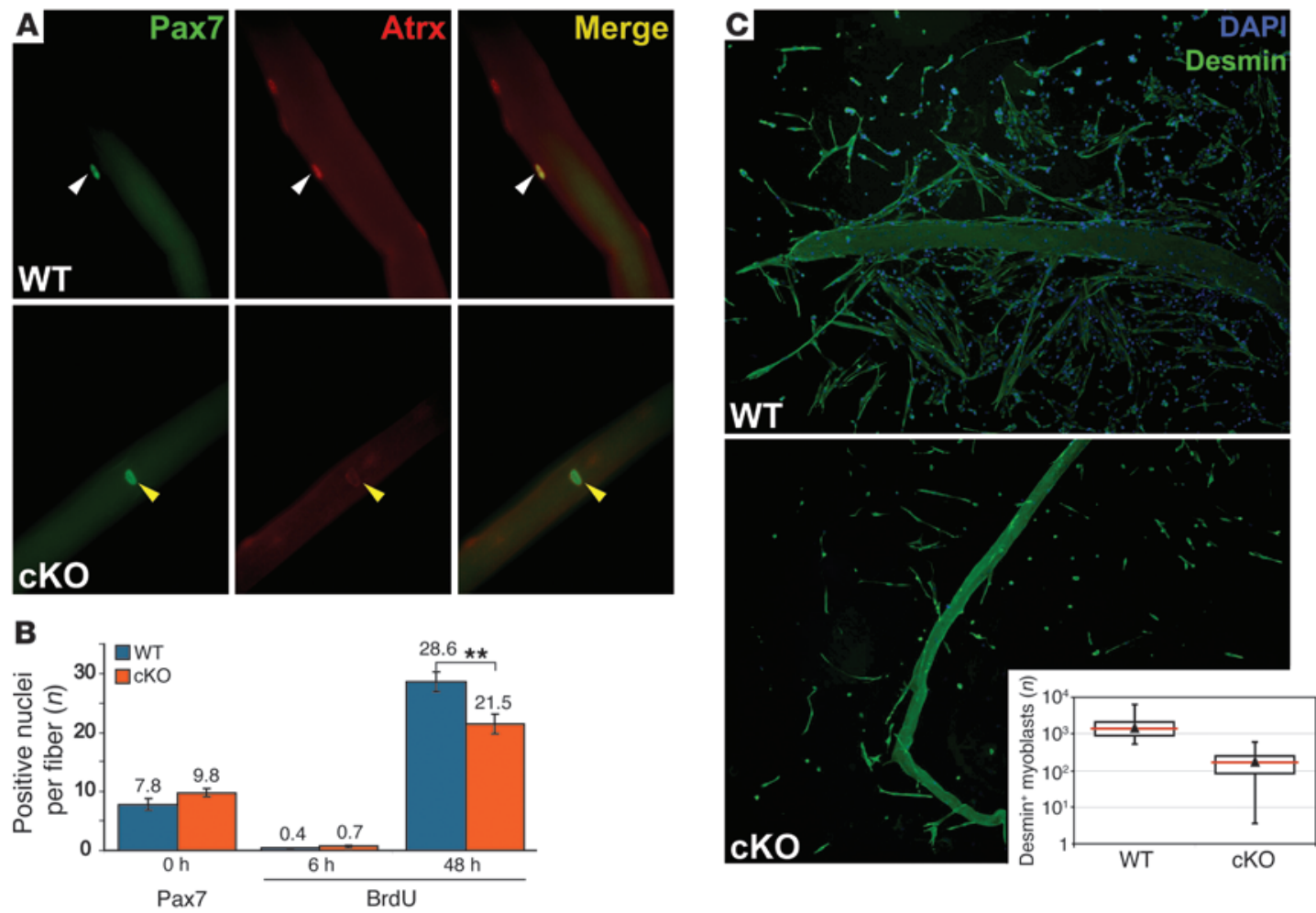

Figure 2

Atrx cKO muscle has normal numbers of satellite cells, yet is unable to efficiently expand the myoblast population when activated in culture. (A) Double-immunofluorescent micrograph of Atrx and Pax7 from freshly isolated Atrx ${ }^{f / y}$ and Atrx cKO EDL myofiber. Pax $7^{+}$satellite cells were still present in Atrx cKO myofibers (yellow arrowheads). (B) Myoblast proliferation index of activated satellite cells from single muscle fiber cultures in suspension. Single muscle fibers isolated from Atrx fly and Atrx cKO EDL muscles were immediately fixed (0 hours) or were grown in BrdUsupplemented GM, then harvested and fixed after 6 and 48 hours in culture. Immunostaining for Pax7 ( 0 hours) and BrdU (6 and 48 hours) was performed, and positive nuclei were enumerated. Values represent mean \pm SEM. Atrx ${ }^{f / y}$, 88 fibers $(n=4)$; Atrx cKO, 66 fibers $(n=3)$. ${ }^{* *} P<0.01$, $t$ test. (C) Myoblast counts from single muscle fibers after 6 days in culture. Single EDL muscle fibers from Atrx ${ }^{f / y}$ and Atrx cKO mice were plated onto a Matrigel substrate and fixed after 6 days in culture. Desmin ${ }^{+}$myoblasts were enumerated for each fiber (inset). Atr ${ }^{f / y}, 58$ fibers $(n=2)$, Atrx cKO, 64 fibers $(n=4)$. Box plots represent quartile distribution of Desmin ${ }^{+}$myoblasts per fiber. Original magnification, $\times 400(\mathbf{A}) ; \times 200(\mathbf{C})$. 
A
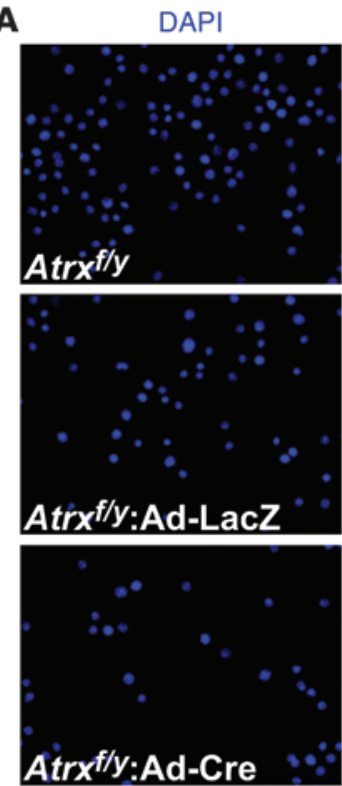

E

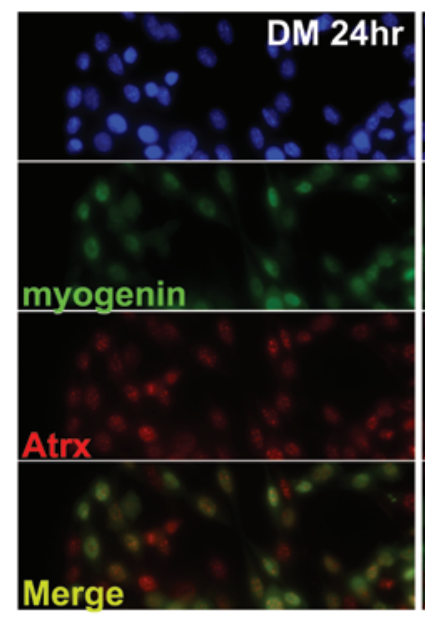

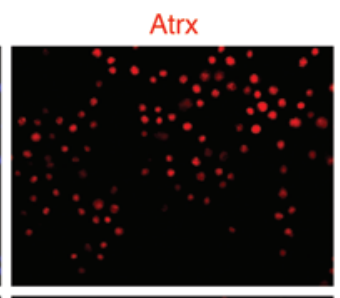
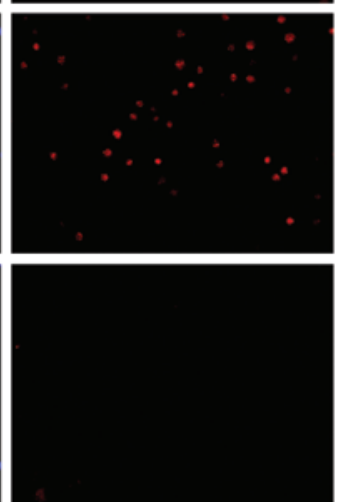

B

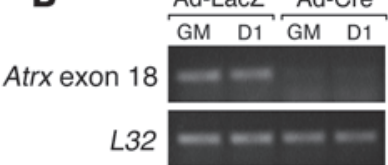

C $\alpha$-Tubulin

D

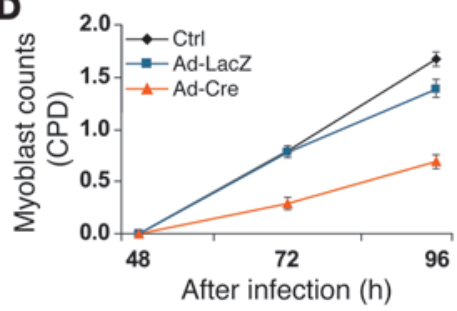

$\mathbf{F}$
Atrx

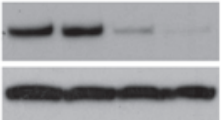

After infection (h)

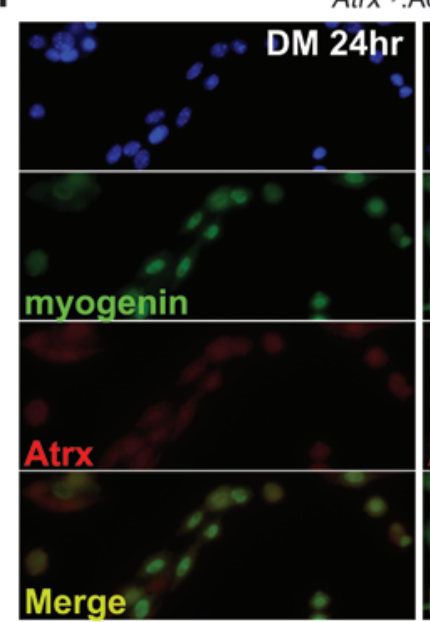

Atrx $^{t y}:$ Ad-Cre

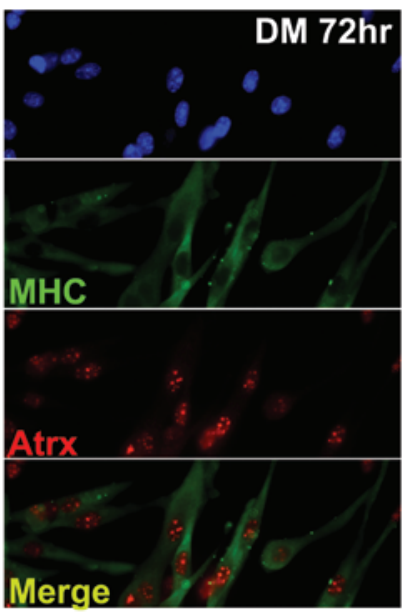

Figure 3

De novo deletion of $A$ trx reduces the expansion rate of myoblasts, but does not affect their capacity to terminally differentiate. (A) Immunostaining for Atrx in uninfected, Atr $x^{f / y}$ :Ad-LacZ, and Atrx ${ }^{f y}$ :Ad-Cre primary myoblasts. (B) RT-PCR analysis of Atrx exon 18 excision after Ad-Cre infection. RNA was harvested from myoblasts in growth conditions (GM) or after 1 day in differentiation media (D1). Ribosomal gene L32 was used as a loading control transcript. (C) Immunoblot analysis of Atrx protein levels after Ad-Cre infection. (D) Cumulative population doubling growth curve of uninfected (Ctrl), Atrx $x^{f / y}:$ Ad-LacZ, and Atrx fly:Ad-Cre myoblasts. Equal numbers of cells were initially seeded for each time point. Cumulative population doublings (CPDs) were obtained from total viable cell numbers at each time point. Error bars represent \pm SEM $(n=4)$. (E and F) Differentiation time course of $A t r x^{f / y}: A d-L a c Z(E)$ and $A t r x^{f / y}$ :Ad-Cre (F) myoblasts. Myoblasts were induced to differentiate in low-mitogen differentiation media (DM) and subsequently fixed after 24 and 72 hours. Differentiation capacity was assessed by immunostaining for the early differentiation marker myogenin and the terminal differentiation marker MHC. Original magnification, $\times 200(\mathbf{A}) ; \times 400(E$ and F).

trols (Figure 2B). Dramatic defects in myoblast expansion became evident when Atrx cKO fibers were left in culture for longer periods. For these experiments, myofibers were adhered to a Matrigel substrate and maintained in culture for 6 days. The muscle-specific intermediate filament protein Desmin was used to mark myoblasts for enumeration. Indeed, Atrx deletion was only evident in Desmin ${ }^{+}$ myoblasts, whereas Desmin ${ }^{-}$fibroblastic cells all expressed Atrx (Supplemental Figure 1C). Quantitative assessment of Desmin ${ }^{+}$ myoblasts confirmed a 9-fold reduction in numbers generated by
Atrx cKO fibers (Figure 2C). On average, the Atrxf/y controls generated 1,700 myoblasts per fiber, compared with 190 from Atrx cKO mice. We therefore concluded that inefficiency of myoblast expansion is the primary cause of the reduction in postnatal limb musculature and the severe regeneration deficit in Atrx cKO mice.

De novo deletion of Atrx does not affect terminal differentiation of myoblasts. The defective growth properties of Atrx cKO myoblasts precluded our attempts to establish primary cultures for further characterization. We therefore used an alternate methodology to 
A
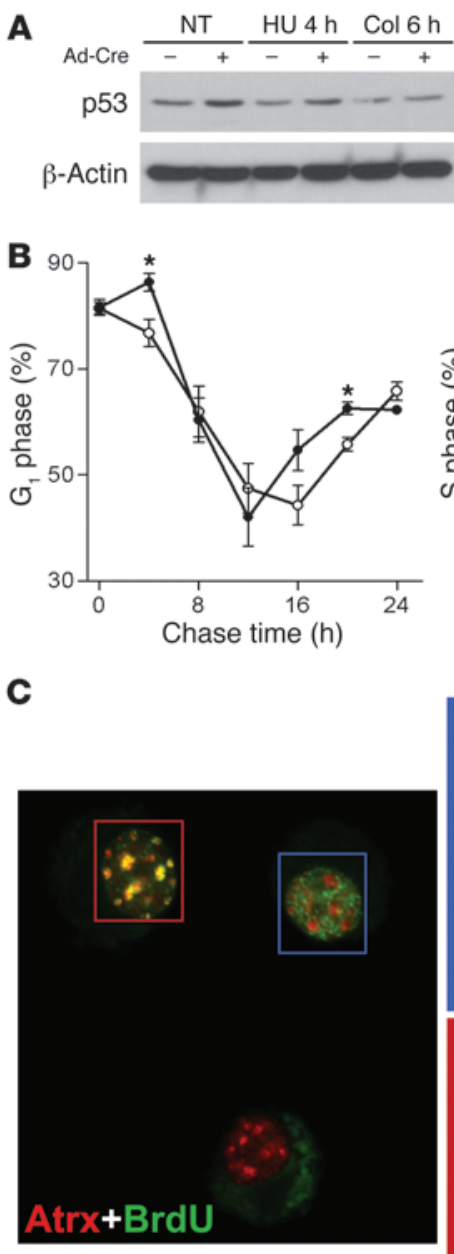
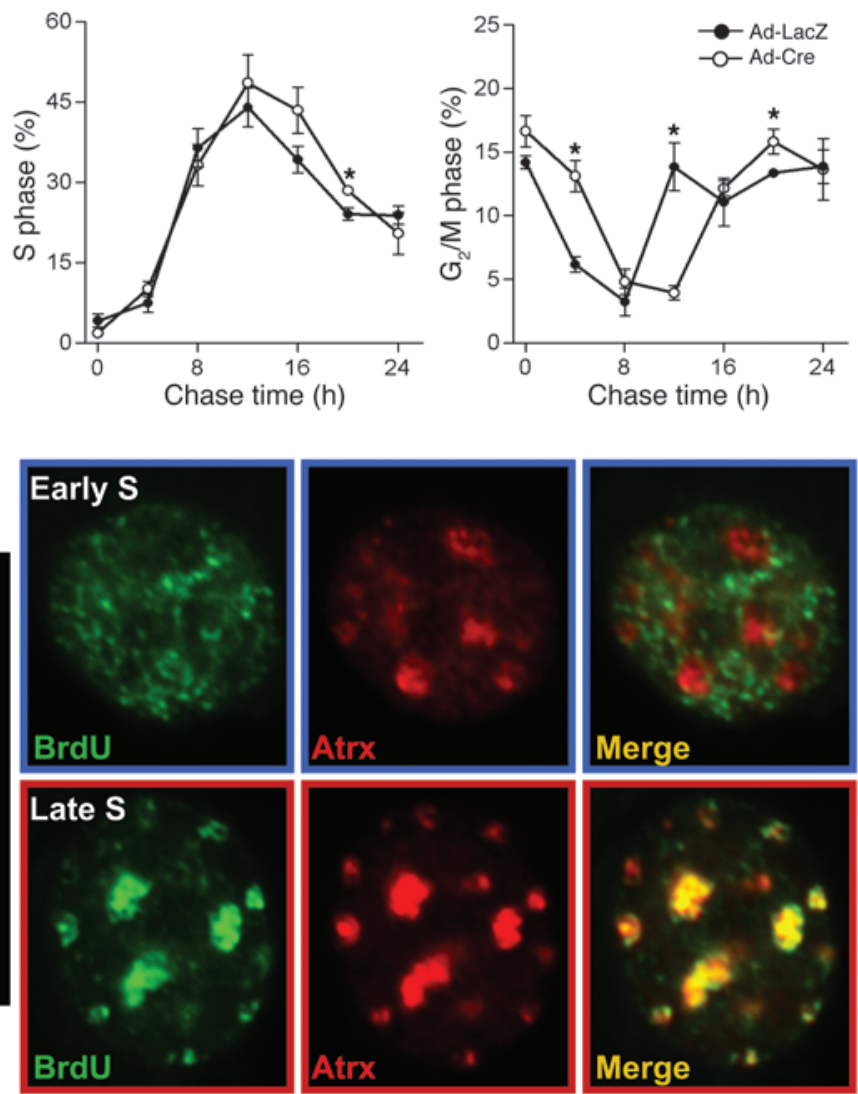

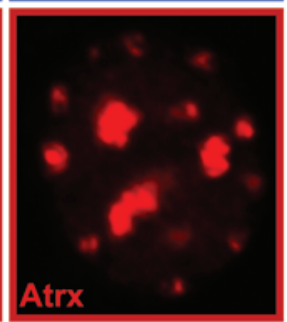

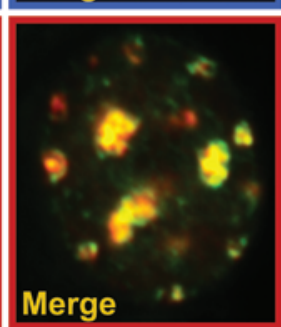

\section{Figure 4}

Atrx depletion delays $S$ phase and induces accumulation of p53 protein. (A) Immunoblot analysis of p53 protein levels after infection with Ad-LacZ (-) or Ad-Cre (+). Infected cells were untreated (NT) or treated with hydroxyurea $(\mathrm{HU})$ or colchicine (Col) for the indicated times, and then protein samples were harvested. $\beta$-Actin was used as loading control. (B) Flow cytometry cell cycle progression analysis of $\mathrm{BrdU}^{-}$population from Atrx $\mathrm{At}^{f / \mathrm{Ad}}$ LacZ and Atrx ${ }^{t / y}$ :Ad-Cre myoblasts. Graphs represent percent BrdUcells in the $G_{1}, S$, and $G_{2} / M$ gates at the indicated time points after the moment of BrdU removal (0 hour). Data are mean \pm SEM $(n=4)$. ${ }^{\star} P<0.05, t$ test. (C) $S$ phase-specific double immunostaining for Atrx and BrdU. Atrx fly myoblasts were pulsed for 20 minutes with BrdU and immediately fixed. Atrx colocalized to late replicating regions of DNA (red box), but did not colocalize with early replicating DNA (blue box). Original magnification, $\times 630(\mathbf{C})$. Boxed regions are enlarged $\times 3$ at right. isolate Atrx-knockout primary myoblasts and study their functional properties. Primary myoblast cultures were established from Atrx $x^{f / y}$ animals and subsequently infected with Cre recombinaseexpressing adenovirus (Ad-Cre). The resulting myoblasts (referred to herein as Atrxf/y:Ad-Cre) exhibited near-complete elimination of Atrx, as assessed by immunofluorescent staining, RT-PCR, and Western blot analysis (Figure 3, A-C).

A slower rate of expansion was clearly evident after infection in Atr $x^{f / y}$ :Ad-Cre myoblasts compared with Ad-LacZ-infected (Atr $x^{f / y}$ : Ad-LacZ) myoblasts and uninfected controls (Figure 3D). Indeed, these results remained consistent with what was observed in the Atrx cKO fiber culture experiments. As such, we used these cells to assess whether loss of Atrx function has an inhibitive effect on myogenic differentiation. Upon withdrawal from high-mitogen growth conditions, Atrxf/y:Ad-Cre myoblasts were capable of fusing to form multinucleated syncytial myotubes. Furthermore, Atr $x^{f / y}$ :Ad-Cre myoblasts were fully capable of inducing the differentiation program, as indicated by the expression of both the early (myogenin) and terminal (myosin heavy chain [MHC]) markers of differentiation (Figure 3, E and F).

Atrx depletion delays $S$ phase and induces accumulation of $p 53$ protein. While previous work has highlighted a growth deficit in Atrx-null ES cells and siRNA knockdown cell culture models, there remains some confusion regarding the underlying mechanism. Proposed mechanisms include enhanced apoptosis, chromatid cohesion and congression defects during mitosis, and defective loading of histone H3.3 during transcription or replication $(3,17,19,20,31)$. To begin to assess the mechanism underlying the poor proliferative capacity of Atrx cKO myoblasts, we first asked whether loss of Atrx function could deregulate the expression of cell cycle genes in our myoblast cultures. 3 biologically independent Atrxf/y myoblast lines were infected with control Ad-LacZ or Ad-Cre virus and subsequently harvested for RNA 48 hours after infection. Quantitative real-time RT-PCR (QPCR) expression analysis detected no deregulation of cell cycle genes such as Ccnd1, Ccne1, Ccna2, Ccnb1, Cdk2, Cdk4, p107, p130, E2f4, and E2f2 at the RNA level. However, we observed 1.3-fold upregulation of the cyclin-dependent kinase (Cdk) inhibitor Cdkn2a, while E2f1, E2f3, and Ccnc were downregulated 1.3-fold, and Ccna1 and Ccnb2 were downregulated 1.5- and 1.3-fold, respectively (Supplemental Figure 2A). Interestingly, protein immunoblot analysis detected changes not seen in our RNA analysis for the cell cycle regulators $\mathrm{p} 107$, cyclin $\mathrm{E}$, cyclin A, and the Cdk inhibitor p27 (Supplemental Figure 2B). In addition to aberrant cell cycle gene regulation, we consistently observed accumulation of $\mathrm{p} 53$ protein in Atrxf/y:Ad-Cre myoblasts (Figure 4A), which suggests that one or more cell cycle checkpoints may be activated in the absence of Atrx. 

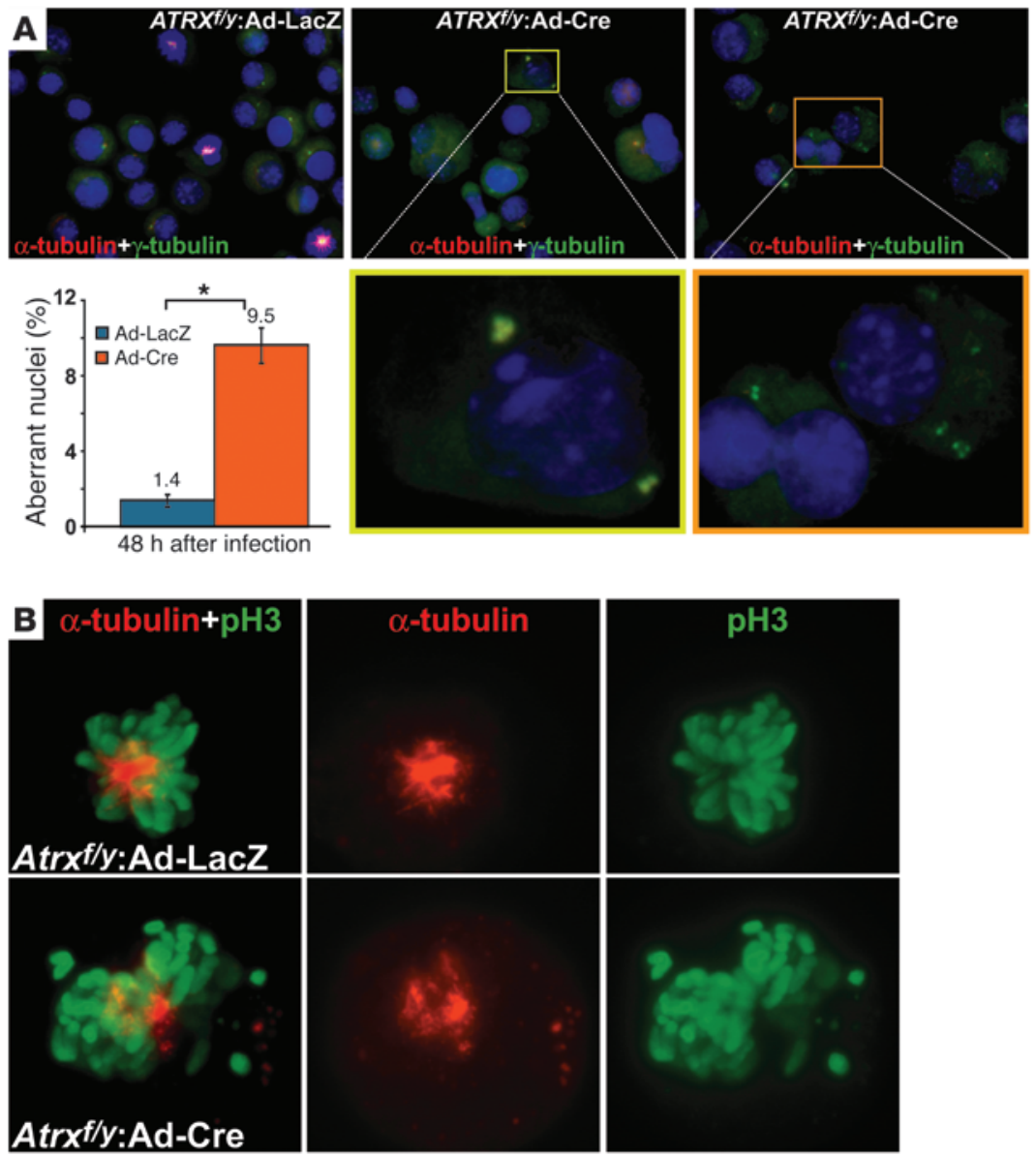

\section{Figure 5}

Loss of Atrx results in genomic instability and aberrant mitotic events. (A) Double-immunofluorescent $\alpha$-tubulin and $\gamma$-tubulin labeling of the mitotic spindle apparatus. Control Atrx fly:Ad-LacZ myoblasts formed well-organized bipolar mitotic spindle assemblies Atrx fly:Ad-Cre myoblasts displayed an unusual cellular phenotype, characterized by a higher frequency of cells with aberrant nuclear morphology and poorly formed mitotic spindles with supernumerary mitotic organizing centers. Also shown is percent cells with aberrant nuclei (binucleated, fragmented micronuclei, or polynuclei) in Atrx ${ }^{f / y}:$ Ad-LacZ and Atrx ${ }^{f / y}:$ AdCre myoblasts. Values represent percent total $\pm 95 \%$ confidence interval. Atrx ${ }^{f / y}$ :Ad-LacZ, 5,116 total cells $(n=3)$; Atrx ${ }^{f / y}$ :Ad-Cre, 4,491 total cells $(n=3)$. ${ }^{*} P<0.05, t$ test. (B) Double-immunofluorescent $\alpha$-tubulin and phospho-histone H3 labeling of the mitotic spindle apparatus and metaphase chromosomes of Atrx ${ }^{f / y}:$ Ad-LacZ and Atrx ${ }^{f / y}$ :Ad-Cre myoblasts. Original magnification, $\times 630(\mathbf{A}$ and $\mathbf{B})$. Boxed regions in $\mathbf{A}$ are enlarged $\times 7$ (yellow) and $\times 4$ (orange) below.

To test whether loss of Atrx elicits a stage-specific cell cycle checkpoint, we treated Atrx $x^{f / y}:$ Ad-Cre myoblasts with the DNA replication inhibitor hydroxyurea or the mitotic inhibitor colchicine. Interestingly, p53 accumulation was observed with hydroxyurea treatment, but not in the presence of colchicine (Figure 4A), which suggests that loss of Atrx triggers the p53 checkpoint during the $S$ phase of the cell cycle. This was a surprising result, as we anticipated that p53 accumulation would be associated with colchicine treatment, as suggested by other studies $(5,31)$. Our results prompted us to specifically examine cell cycle kinetics in Atrx $x^{f / y}$ :Ad-Cre myoblasts by flow cytometry. A static 1-parameter analysis of asynchronous populations failed to detect any effect of Atrx loss on cell cycle distribution, consistent with work in Atrx-null ES cells (27). To determine whether Atrx loss results in a more subtle defect in cell cycle progression, a BrdU pulse-chase protocol was used (32). Briefly, Atr $x^{f} / y$ :Ad-LacZ and Atr $x / y$ :Ad-Cre myoblasts were pulsed with BrdU for 1 hour and subsequently collected at 4-hour intervals for 2-parameter flow cytometric analysis. The BrdU pulse served to exclude the $S$ phase population, delineating a $\operatorname{BrdU}^{-} \mathrm{G}_{1}$ and $\mathrm{G}_{2} / \mathrm{M}$ population at the 0 -hour time point (immediately following the 1-hour BrdU pulse). Cell cycle progression from 0 to 24 hours was assessed in the $\mathrm{BrdU}^{-}$population as they transited from $\mathrm{G}_{1}$ through $\mathrm{S}$ and into $\mathrm{G}_{2} / \mathrm{M}$. Approximately $85 \%$ of the $\mathrm{BrdU}^{-}$cells were in the $\mathrm{G}_{1}$ phase of the cell cycle at the 0 -hour time point, while the remainder was in $\mathrm{G}_{2} / \mathrm{M}$ (Figure $4 \mathrm{~B}$ ). Orderly passage of the control cells from $G_{1}$ and $G_{2} / M$ through the cell cycle occurred during the chase period, while a small but statistically significant delay in the pas- sage of $A t r x f / y$ :Ad-Cre myoblasts from $\mathrm{G}_{1}$ through $\mathrm{S}$ and $\mathrm{G}_{2} / \mathrm{M}$ into the subsequent $\mathrm{G}_{1}$ phase of the cell cycle was observed (Figure $4 \mathrm{~B}$ ). These results indicated that the absence of Atrx delayed the completion of S phase and consequently produced a transient disequilibrium in the cell cycle profile at the 16- and 20-hour time points (Supplemental Figure 3). Similarly, the passage of the $\mathrm{G}_{2} / \mathrm{M}$ population of $\mathrm{BrdU}^{-}$Atrx $x^{f / y}$ :Ad-Cre myoblasts was delayed compared with Atrx $x^{f / y}:$ Ad-LacZ controls (Figure 4B). In light of these findings, we examined Atrx localization with respect to sites of DNA replication during $\mathrm{S}$ phase. Active sites of DNA replication were marked with a brief 20 -minute BrdU pulse in asynchronously proliferating myoblasts. Coimmunolocalization revealed a strikingly similar pattern between late-replicating chromatin domains and Atrx (Figure $4 \mathrm{C}$, red box). These lines of evidence support a putative function for Atrx in the processing of late-replicating chromatin.

Loss of Atrx results in genomic instability, fragile telomeres, and aberrant mitotic events. Replication errors frequently lead to chromosomal abnormalities that affect native chromatin structure and associated functions. Atr $x^{f / y}:$ Ad-Cre myoblasts presented an abnormal cytologic phenotype characterized by an increase in irregular nuclear morphology, poorly formed mitotic spindles, binucleation of cells, and supernumerary $(>2) \gamma$-tubulin-staining microtubule organizing centers (Figure 5A). Quantification of the proportion of myoblasts with aberrant nuclear morphology (micronuclei, binuclei, or multinuclei) revealed a significantly higher percentage in Atrxf $f^{f}$ :AdCre than in Atrxf/y:Ad-LacZ myoblasts (Figure 5A). Furthermore, visualization of the mitotic spindle revealed substantial contrast 

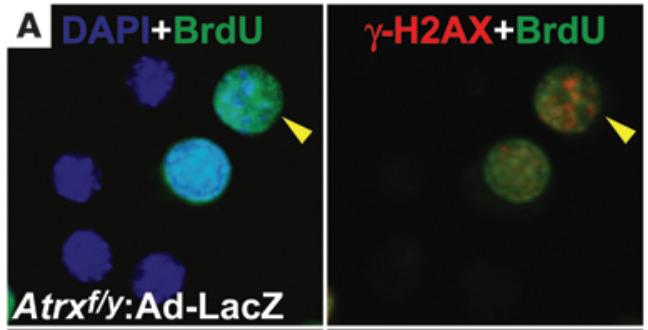

B
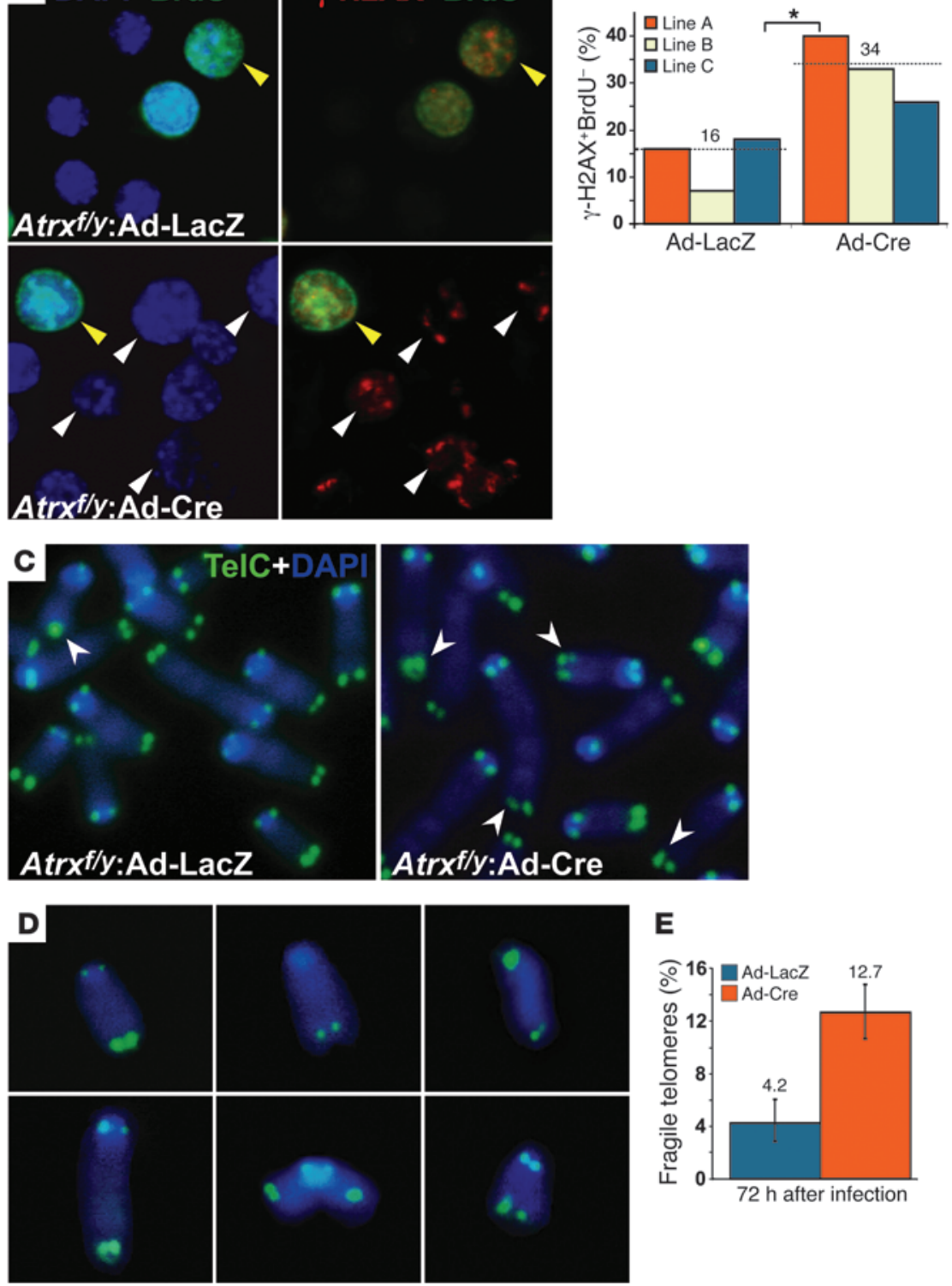

E

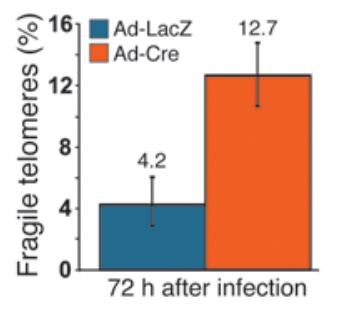

\section{Figure 6}

Atrx inhibits DNA damage-induced $\gamma-\mathrm{H} 2 \mathrm{AX}$ foci formation and telomere fragility. (A) Doubleimmunofluorescent staining for BrdU and $\gamma-\mathrm{H} 2 \mathrm{AX}$ of Atrx ${ }^{f / y}$ :Ad-LacZ and Atrx ${ }^{f / y}$ :Ad-Cre myoblasts. Yellow arrowheads, $\gamma-\mathrm{H} 2 \mathrm{AX}+\mathrm{BrdU}^{+}$nuclei; white arrowheads, $\gamma-\mathrm{H} 2 \mathrm{AX}+\mathrm{BrdU}-$ nuclei. (B) Percent $\gamma-\mathrm{H}_{2} \mathrm{AX}+\mathrm{BrdU}^{-}$foci in Atr ${ }^{f / y}$ :Ad-LacZ and Atrx ${ }^{f / y}$ : Ad-Cre myoblasts. Values for Ad-LacZ (15.8\%) and Ad-Cre (33.8\%) were calculated from infection of 3 biologically independent primary myoblast lines. ${ }^{*} P<0.05, t$ test. (C) Fluorescence (FITC) labeling of telomeres on condensed metaphase chromosomes in Atrx ${ }^{f / y}:$ Ad-LacZ and Atrx ${ }^{f / y}$ :Ad-Cre myoblasts. Arrowheads denote chromosomes with aberrant telomeric signals. (D) Representative images of normal (top left), deleted (top middle), merged (top right), bridged (bottom left), duplicated (bottom middle), and diffused (bottom right) telomeric signals. (E) Percent fragile telomeres in mitotic Atrx ${ }^{f / y}: A d-L a c Z$ and Atr $X^{f / y}$ :Ad-Cre myoblasts. Values represent percent total $\pm 95 \%$ confidence interval. Atrx ${ }^{t / y}$ :AdLacZ, 661 chromosomes $(n=3)$; Atrx ${ }^{f / y}$ :Ad-Cre, 1,096 chromosomes $(n=3)$. Original magnification, $\times 630(\mathbf{A}) ; \times 1,000(\mathbf{C}$ and $\mathbf{D})$. between control Atrxf/y:Ad-LacZ and Atrxf/y:Ad-Cre myoblasts. In mitotic Atr $x^{f} y$ :Ad-LacZ myoblasts, $\alpha$-tubulin staining of the spindle fibers revealed a well-formed and organized spindle apparatus (Figure 5B). However, despite possessing a bipolar organizing center, $\alpha$-tubulin staining of the spindle fibers was weaker and more disorganized in mitotic Atrxf/y:Ad-Cre myoblasts. The defect in mitotic spindle formation was also associated with a scattered alignment of metaphase chromosomes (Figure 5B). Interestingly, visualization of phospho-histone H3-stained metaphase chromosomes revealed what appeared to be fragmented microchromosomes (Figure 5B), indicative of genomic instability. The presence of genomic instability prompted us to examine the potential activation of DDR pathways by $\gamma$-H2AX staining (Figure 6A). The percentage of cells with $\gamma$-H2AX foci were significantly increased in Atr $x^{f / y}$ :Ad-Cre versus Atr $x^{f / y}$ :Ad-LacZ myoblasts (Figure 6B).

Atrx is highly enriched in PML bodies and pericentromeric heterochromatin (33). Moreover, recent studies have revealed an association between histone variant $\mathrm{H} 3.3$ and Atrx on telomeres in mouse ES cells $(17,19)$. Dysfunctional centromeres and telomeres often lead to a multitude of cellular responses, such as chromosomal instability, activation of cell cycle checkpoints, and hyperactivated DDR (34-36). Inability to reestablish function of these crucial structures can result in mitotic catastrophe and eventually cell death. We therefore examined the telomeres and centromeres of Atrx $f / y$ :Ad-Cre myoblasts for evidence of dysfunction. Visualization of the telomeres using FISH revealed an increased frequency of aberrant telomeric signals in Atrxf $f^{f} y$ :Ad-Cre myoblasts (Figure 6C). Telomeric aberrations (fragile telomeres) were scored as deletions, merged, duplicated, bridged, or diffuse telomeric signals (Figure $6 \mathrm{D})$. Under these criteria, we observed a 3-fold increase in the frequency of fragile telomeres in Atrx f/y:Ad-Cre versus Atrx f/y:Ad-LacZ myoblasts (Figure 6E and Table 2). Despite the clear increase in the incidence of fragile telomeres, we were unable to detect higher levels of centromeric damage by $\gamma$-H2AX and CENP-A costaining 
Table 2

Fragile telomere classification

\begin{tabular}{lccccc} 
& Loss & Gain & Diffuse & Bridge & Total \\
Atrx $x^{f y}:$ Ad-LacZ & 11 & 8 & 7 & 2 & 661 \\
Atrx $y$ :Ad-Cre & 72 & 25 & 20 & 22 & 1,096 \\
\hline
\end{tabular}

Values denote number of chromosomes per classification $(n=3$ experiments per group).

(Supplemental Figure 4). Taken together, these data demonstrate that proliferating Atr $x^{f / y}$ :Ad-Cre myoblasts accumulate DNA damage, and thus suggest that Atrx is involved in the processing or maintenance of late-replicating heterochromatin.

Activation of ATM and $p 53$ is concomitant with reduction of Rad51 in Atrx-depleted myoblasts. Properly functioning DDRs are critical for maintaining genomic stability during the cell cycle. ATM protein kinases, along with ATM- and Rad3-related (ATR) protein kinases, are activated in response to replication stress caused by collapsed or stalled DNA replication forks (37). We therefore assessed ATM/ ATR signaling in Atrx-depleted myoblasts to determine the presence of excessive replicative stress.

Western blot analyses of Atrxf/y:Ad-Cre myoblasts revealed an average 3 -fold increase in phospho-ATM ${ }^{\text {Ser1981 }}$ and a 1.5 -fold increase of phospho-Chk $1^{\text {Ser345 }}$, an ATR kinase substrate (Figure 7A and Supplemental Table 2). Activation of ATM simultaneously targets downstream effectors such as p53, while mobilizing the homologous recombination (HR) repair pathway. HR repair proteins, such as Brca1 and Rad51, are essential for proper $S$ phase progression and maintenance of genomic stability. Consistent with our earlier observation, p53 protein was increased approximately 2 -fold in Atrxf ${ }^{f}$ : Ad-Cre myoblasts (Figure 7A and Supplemental Table 2). Interestingly, levels of the HR repair protein Rad51 were reduced by 2-fold in $A \operatorname{tr} x^{f / y}$ :Ad-Cre compared with Atr $x^{f / y}$ : Ad-LacZ myoblasts (Figure $7 \mathrm{~B}$ and Supplemental Table 2). We further examined the capacity of proliferating myoblasts to recover from acute exposure to UV-induced DNA damage in the absence of Atrx. Atr $x^{f / y}$ :Ad-Cre and Atr $x^{f / y}$ :Ad-LacZ myoblasts were pulsed with $10 \mathrm{~J} / \mathrm{m}^{2} \mathrm{UV}$ and harvested 24, 48, and 72 hours later. Under high-mitogen growth conditions, Atr $x^{f / y}$ :Ad-Cre myoblasts maintained elevated levels of both p53 and the DNA damage marker $\gamma$-H2AX 72 hours after UV irradiation (Figure 7C). In contrast, Atr $x^{f / y}$ :Ad-LacZ controls showed recovery from UV irradiation by 48 hours with p53, and $\gamma$-H2AX levels diminished to very low levels by 72 hours. Finally, we further validated the intrinsic requirement for Atrx in rapidly proliferating myoblasts by adenoviral reintroduction of ATRX (Ad-ATRX) into Atr $x^{f / y}$ :Ad-Cre myoblasts (Supplemental Figure 5). After AdATRX infection, we observed reduced levels of activated Chk1 compared with Atrx $x^{f / y}$ :Ad-Cre myoblasts, although p53 levels remained elevated and the percent total of aberrant nuclei

\section{Figure 7}

was not significantly reduced (Supplemental Figure 5, A and B). It is likely that significant chromatin changes occur in the 48 hours prior to ATRX reintroduction that dampen more robust rescue of the aberrant nuclei. Nonetheless, we did observe a $36 \%$ increase in the number of viable cells after Ad-ATRX infection (Supplemental Figure 5C), demonstrating partial rescue of the growth defect.

ATRX knockdown in buman primary myoblasts results in growth deficits and p53 accumulation. Due to a high degree of homology between mouse and human ATRX (6), one would expect satellite cell expansion deficiencies to be similar in the absence of functional ATRX in humans. In order to validate this assumption, we transfected human primary myoblasts with either nonspecific control siRNA (siScrambled) or ATRX-specific siRNA (siATRX) (Figure 8, A and B). Western blot analysis demonstrated an increase in p53 protein levels in siATRX-knockdown myoblasts compared with siScrambled controls (Figure 8B). Moreover, loss of ATRX in human primary myoblasts significantly reduced proliferation, as determined by a $36 \%$ reduction in WST-1 cleavage by mitochondrial dehydrogenases (Figure 8C). We also examined a panel of 3 ATR-X patientderived lymphoblast lines harboring different mutations in ATRX (Figure 8D). Line 1 contained a 2 -kb deletion spanning the int $34 /$ ex35 junction, resulting in a short C-terminal truncation; line 2 contained a Gly249Cys substitution in the ADD domain; and line 3 carried a $100-\mathrm{kb}$ deletion spanning exons $2-7$, producing an internally deleted protein lacking a functional ADD domain that was detectable by a C-terminal specific Atrx antibody (M.S. Huh, unpublished observations). Immunoblot detection using a monoclonal antibody directed toward an N-terminal epitope of ATRX detected signals in both UV-treated and untreated controls as well as patient lines 1 and 2 (Figure 8D). Importantly, we observed in
A

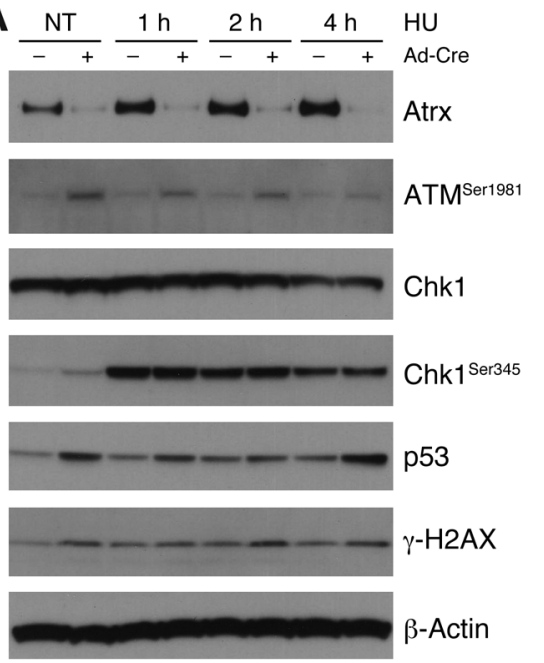

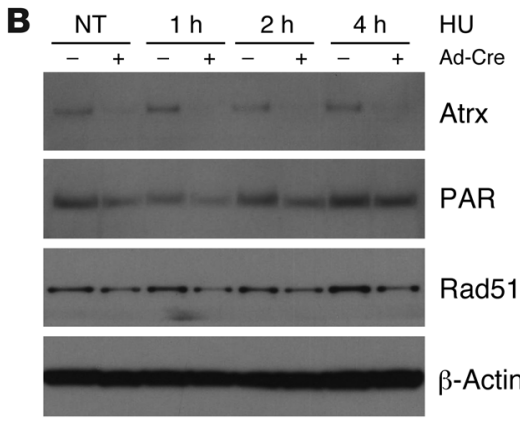

C

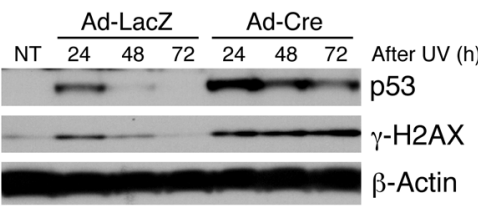

Atrx depletion activates the p53-ATM DDR pathway and concomitant reduction in Rad51. (A and B) Western blot analysis of Atrx ${ }^{f / y}:$ Ad-LacZ (-) and Atrx fly:Ad-Cre (+) myoblasts exposed to the replication inhibitor hydroxyurea. (A) Immunoblots were probed with anti-

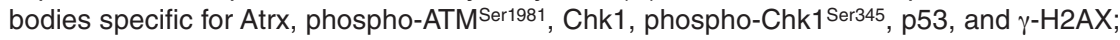
$\beta$-actin was used as loading control. (B) Immunoblots were probed with antibodies specific for Atrx, PAR, and Rad51; $\beta$-actin was used as loading control. (C) Western blot analysis of UV-induced DDR in Atr $x^{f / y}$ :Ad-LacZ and Atrx $f / y$ :Ad-Cre myoblasts under high-mitogen growth conditions. Infected myoblasts were left untreated or were irradiated ( $10 \mathrm{~J} / \mathrm{m}^{2} \mathrm{UV}$ pulse), and protein was harvested at 24,48 , and 72 hours. Immunoblots were probed with antibodies specific for p53 and $\gamma$-H2AX; $\beta$-actin was used as loading control. 
A

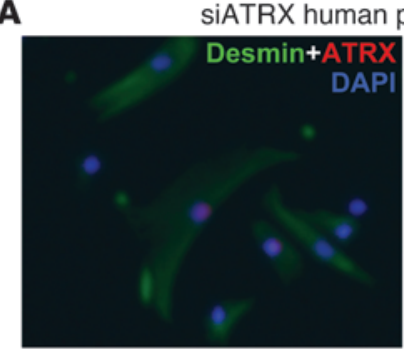

B

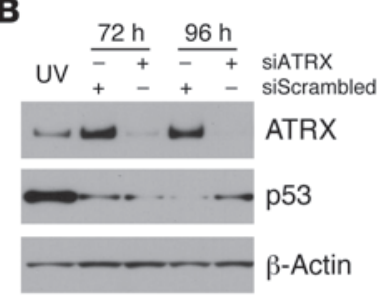

D

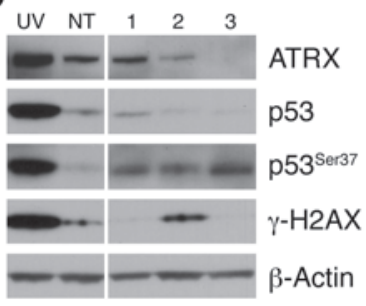

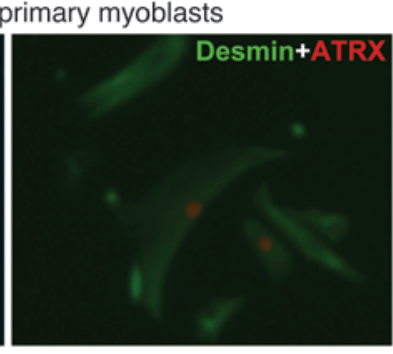

C

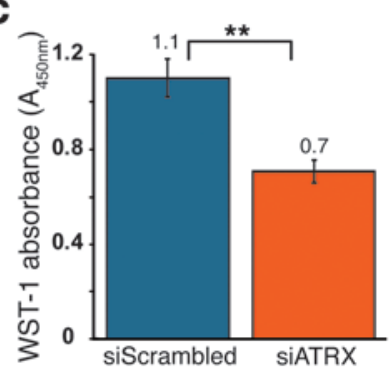

\section{Figure 8}

ATRX knockdown in human primary myoblasts leads to p53 induction and reduced proliferative capacity. (A) Double-immunofluorescent staining for skeletal muscle-specific intermediate filament proteins Desmin and ATRX 72 hours after transfection with siATRX. (B) Western blot analysis of human primary myoblasts transfected with siScrambled and SiATRX. Immunoblots were probed with antibodies specific for ATRX and p53; $\beta$-actin was used as loading control. (C) WST-1 proliferation assay. Values represent mean $\pm \operatorname{SEM}(n=4)$. ${ }^{\star \star} P<0.01, t$ test. (D) Western blot analysis of immortalized ATRX mutant patient lymphoblast lines (numbered 1-3). UV, $10 \mathrm{~J} / \mathrm{m}^{2} \mathrm{UV}$ irradiation of WT lymphoblasts; NT, untreated WT lymphoblasts. Immunoblots were probed with antibodies specific for ATRX, p53, phosphorylated $\mathrm{p} 53^{\mathrm{Ser} 37}$, and $\gamma-\mathrm{H} 2 \mathrm{AX} ; \beta$-actin was used as loading control. Lanes were run on the same gel but were noncontiguous (white lines). Original magnification, $\times 200$ (A).

sequently, the developmental growth of embryonic and later fetal muscle tissue is dependent on a different population of progenitor cells that are $\operatorname{Pax}^{+} \mathrm{Pax}^{+}(44,45)$. Moreover, Relaix et al. demonstrated that $93 \%$ of $\mathrm{Pax}^{+} \mathrm{Pax}^{+}$muscle progenitors were Myf5-, undergoing massive proliferation prior to MRF expression and differentiation (44). Consequently, the developmental inactivation of Atrx in our Atrx cKO model occurred after the rapid expansion of $\mathrm{Pax}^{+} \mathrm{Pax} 7^{+}$progenitors, resulting in viable offspring.

In the first 3 weeks of life, muscle mass accumulates rapidly through the accretion of myonuclei from activated muscle satellite cells in the mouse $(46,47)$. Despite their small size, Atrx cKO mice had normal numbers of resident satellite cells that were capable of activating and entering a proliferative phase. However, fewer myoblasts were evident within 48 hours, and profound deficiencies in myoblast expansion were realized after culturing fibers for 6 days. It was dramatically evident from the final numbers that Atrx-null myoblasts were incapable of sustaining a rapid growth rate. Consequently, under natural physiological growth demands, muscle mass accumulation was delayed in Atrx cKO mice. Beyond 3 weeks to full adulthood, addition of myonuclei ceases, and muscle mass increase is achieved through hypertrophic growth of the muscle fiber (47). It was during this period that we observed the gradual compensation of body mass in Atrx cKO mice compared with their Atrxofy littermates.

Activation and expansion of satellite cell-derived myoblasts is also fundamental for proper muscle regeneration after exercise or injury (48). CTX ablation of muscle is an extreme experimental paradigm that destroys differentiated muscle fibers and $90 \%$ of satellite cells, thus placing an extensive demand on the remaining satellite cells for regeneration $(29,49)$. Our analysis of CTX-injected Atrx cKO muscle demonstrated a profound deficit in regeneration, as revealed by poorly formed residual microfibers at the site of injury in addition to large vacuolar spaces and a high degree of fibrosis. The fatty/fibrotic phenotype is most likely a direct result of a combination of poor satellite cell expansion and increased compensation by the resident fibro/adipogenic progenitors that expand and differentiate to fill the void (50). Our findings further demonstrated the requirement for Atrx in the hyperexpansion of myogenic progenitor cells.

Delayed $S$ phase progression activates the DDR and leads to genomic instability. It has been previously documented that Atrx-null cells have growth deficiencies, yet the precise mechanism underlying this deficit remained elusive. In particular, a competitive coculture assay between WT and Atrx-null ES cells demonstrated a precipitous disappearance of the Atrx-null population after 4 passages, 
despite the lack of any indication of a defective cell cycle profile (27). Other studies using Atrx shRNA treatment of HeLa cells or a Sertoli cell-specific Atrx conditional knockout mouse described a delay in $\mathrm{G}_{2} / \mathrm{M}$ progression along with increased numbers of dying cells and cells with chromosomal defects (e.g., micronuclei and DNA bridges; refs. 4, 31). Our present results implicate a role for Atrx in the progression through mid to late $\mathrm{S}$ phase, due to the following 2 lines of evidence: (a) in actively dividing Atrxf/y myoblasts, the localization pattern of Atrx was almost indistinguishable from that of late-replicating heterochromatin; and (b) flow cytometry cell cycle chase analysis revealed a distinct and transient delay going through mid to late $\mathrm{S}$ phase by Atr $x^{f / y}$ :Ad-Cre myoblasts. It must be noted that the subtle and transient nature of this phenotype cannot be detected by a static cell cycle profile of asynchronously dividing cells.

Together, these data suggest that Atrx is involved in the replication of heterochromatin and/or its structural reestablishment after DNA synthesis. Moreover, the proper assembly and maintenance of centromeric and/or telomeric heterochromatin is critical for genomic integrity and for cell survival $(5,51-54)$. Under conditions of significant growth, the probability of acquiring deleterious genomic lesions in Atrx-deficient cells is increased, and at some point, cell survival is compromised. This has previously been illustrated by forebrain-specific inactivation of Atrx, which yielded a 12 -fold increase in $\mathrm{TUNEL}^{+}$cells at E11.5 (3). This developmental time point coincides with the moment when neural progenitors are undergoing rapid expansion. Similarly, at E17.5, when Sertoli cells are at their proliferative peak, a dramatic 40-fold increase in apoptosis was observed (4). Results from WT and Atrx-null ES cell culture experiments can be interpreted in a similar manner. Indeed, this may be a common feature of many chromatin remodelling proteins, as similar observations have been made for ISWI, SWI/SNF, and Mi-2 complexes (55-58). Consistent with a role for Atrx in genomic stability, we also observed a higher incidence of $\gamma$-H2AX staining, p53 accumulation, and activation of the ATM damage pathways in Atrx $x^{f / y}$ :Ad-Cre myoblasts that translated into an abnormal cytologic phenotype. These abnormalities were most apparent during mitosis, which suggests that previous studies describing mitotic progression deficits in HeLa and Sertoli cells may have occurred secondary to problems in heterochromatin replication/assembly, as indicated by our experiments.

Involvement of Atrx in telomere replication. Atrx binding to pericentromeric heterochromatin is well documented, and recent studies have determined that this targeting is mediated through recognition of a bipartite histone mark $\left(\mathrm{H} 3 \mathrm{~K} 4 \mathrm{me}^{0} / \mathrm{H} 3 \mathrm{~K} 9 \mathrm{me}^{3}\right)$ via the ADD domain (7-9). Surprisingly, the centromeres of Atrx f/y:Ad-Cre myoblasts remained intact, including HP1 $\alpha$ localization at these structures (M.S. Huh, unpublished observation). A mounting body of recent evidence has suggested a functional role for Atrx at the telomere. In mouse ES cells, chromatin IPs using Atrx antibodies achieved a dramatic enrichment for telomeric repeats (20), and Atrx-Daxx complexes were demonstrated to be essential for the deposition of histone variant $\mathrm{H} 3.3$ onto telomeres $(17,19,59)$. In Atrx-depleted mouse primary myoblasts, we observed telomeric aberrations characterized by a higher incidence of telomeric bridging, duplications, and merging. Loss of telomeric integrity is commonly associated with the disruption of processes involving replication and protection of telomeres (60), and G-rich telomeric tracts are particularly susceptible to the formation of G4 structures (61). Furthermore, chemical stabilization of G4 structures by RHPS4 disrupts telomeric replication, leading to telomeric dysfunction and genomic instability (62). Interestingly, Atrx has been shown to have an affinity for G4 structures in vitro (20). Thus, one could envision Atrx acting to alleviate these G4 structures in an ATPasedependent manner during telomere replication. However, further studies are required to determine whether there is a higher prevalence of G4 structures in the absence of Atrx.

The ATPase domain of Atrx is most similar to that of the HR protein Rad54, which suggests that Atrx may be involved in resolving stalled replication forks (6). The higher frequency of replisome stalling in heterochromatin necessitates the recruitment of homologydirected DNA repair proteins ( $\operatorname{Rad} 51, \mathrm{Brca} 2, \operatorname{Rad} 52$, and $\mathrm{Rad} 54)$ in order to resume DNA replication in an error-free manner (63). In this regard, Atrx and Rad54 are both capable of remodeling mononucleosomes as well as exhibiting a translocase activity $(10,11)$. Despite these similarities, it is unknown whether Atrx can stabilize Rad51 onto presynaptic filaments or resolve DNA intermediate structures (Holliday junctions or stalled replication forks) by driving branch migration, as has been demonstrated for Rad54 (64-67). Nonetheless, our results are congruent with a model proposed by Karlseder and colleagues that demonstrates the recruitment of the MRN complex, ATM kinase, and HR machinery to the newly replicated telomere for the reformation of the protective t-loop $(68,69)$. Coincidently, our results showed that loss of Atrx caused a delay in mid to late S phase that was concurrent with ATM activation and Rad51 reduction. As such, Atrx may provide a Rad54-like activity in promoting the stability of Rad51 on telomeric overhangs, thus facilitating the formation of the protective t-loop.

We demonstrated here that Atrx deficiency in the mouse model was applicable in a clinical context. Much like in mouse myoblasts, loss of ATRX in human myoblasts caused proliferative deficiency and p53 accumulation. Furthermore, we have shown that lymphoblasts from ATR-X patients induced the ATM/ATR DDR. Patient mutations are far less severe than the Atrx knockout model, which validates our animal model. In addition, these lines of evidence support a model in which intrinsic deficiencies in human satellite cells lead to muscle weakness, giving rise to hypotonia, kyphosis, and ambulation difficulties, which are all common features of ATR-X patients.

Our studies have defined the physiological and cellular consequences of Atrx dysfunction in skeletal muscle. More importantly, we highlighted the importance of Atrx in maintaining genomic stability and telomeric integrity in rapidly proliferating myoblasts. These findings support a general function for Atrx in rapidly proliferating progenitor cells.

\section{Methods}

Mice and CTX injury protocols. Myf5-Cre mice were obtained from P. Soriano (Mount Sinai School of Medicine, New York, New York, USA; ref. 26) and maintained on a 129/Sv background. Atrx exon 18 floxed mice (3) were maintained on a C57BL/6 background. See Results for the breeding strategy. CTX regeneration experiments were performed as previously described (70). TA muscles of adult mice were injected with $25 \mu \mathrm{l}$ of $10 \mu \mathrm{M}$ CTX (Latoxan). Muscles were harvested 1 and 2 weeks after injection. H\&E and Masson's Trichrome histology were performed at the University of Ottawa pathology laboratory.

Primary myoblast and single fiber isolation protocols. Primary myoblasts were isolated from hind limbs of 4-week-old Atrx floxed male mice as previously published (39). Primary myoblasts were maintained in Hams F-10 (Wisent) growth media (GM) supplemented with $2.5 \mathrm{ng} / \mathrm{ml}$ human recombinant bFGF (Invitrogen), 20\% FBS (PAA), and 2\% penicillin/streptomycin (Invitrogen). 
Ad-LacZ and Ad-Cre were used at an MOI of 10 on Atrxf/y primary myoblasts, as described previously (71), and Ad-ATRX was used at an MOI of 1.

Live myofibers were dissociated and cultured from the EDL muscle (72). Floating muscle fiber cultures were maintained in $60-\mathrm{mm}$ bacterial plates (Fisher) coated with horse serum (Invitrogen) in DMEM (Invitrogen) supplemented with $10 \%$ horse serum, $0.5 \%$ chick embryo extract (catalog no. CE650T; Accurate Chemical and Scientific Corp.), and $10 \mu \mathrm{M}$ BrdU (Sigma-Aldrich). For adherent fiber cultures, individual fibers were seeded in 8-well chamber slides (catalog no. 177445; Lab-Tek) coated with Matrigel (catalog no. 40234; Becton Dickinson) and maintained in DMEM supplemented with $10 \%$ horse serum and $0.5 \%$ chick embryo extract.

Immunostaining and Western blots. Primary myoblasts were trypsinized and resuspended in GM. Myoblasts were cytospun (Cytospin4; Thermo Shandon) onto slides and fixed in 2\% PFA or ice-cold methanol/acetone at a 1:1 ratio. For immunostaining, myoblasts were permeabilized with $0.1 \%$ Triton- $X$ in PBS and incubated in blocking solution (20\% horse serum, $0.1 \%$ fetal bovine serum, and $0.03 \%$ sodium azide in PBS) for 2 hours at room temperature. Primary antibodies were diluted in PBS and incubated overnight at $4{ }^{\circ} \mathrm{C}$. Myoblasts were stained with antibodies to Pax7 (1:3 dilution; Developmental Studies Hybridoma Bank [DSHB]), Atrx (1:500 dilution, H-300; Santa Cruz), Desmin (1:500 dilution, clone D33; Dako), myogenin (1:10 dilution, F5D; DSHB), MHC (1:20 dilution, MF20; DSHB), BrdU (1:500 dilution, clone B44; BD Biosciences), $\alpha$-tubulin (1:1,000 dilution, clone DM1A; Sigma-Aldrich), $\gamma$-tubulin (1:500 dilution, ab11317; Abcam), pH3 (1:500 dilution, 06-570; Millipore), $\gamma$-H2AX (1:250 dilution, 20E3; Cell Signaling), and CENP-A (1:500 dilution, C51A7; Cell Signaling). Images were taken using either an Axio Imager M1 (Zeiss), LSM 510 (Zeiss), or DMI6000B (Leica) microscopy system.

For Western blots, primary myoblasts were harvested and lysed in RIPA extraction buffer $(50$ mM Tris- $\mathrm{HCl}$, pH 7.4; $1 \%$ Nonidet P-40; $0.5 \%$ sodium deoxycholate; $0.1 \%$ sodium-dodecyl-sulphate; $5 \mathrm{mM} \mathrm{EDTA} ; 150 \mathrm{mM} \mathrm{NaCl}$; and $50 \mathrm{mM} \mathrm{NaF}$ ) supplemented with protease inhibitors (cOmplete mini; Roche) on ice. Protein lysates were separated on a $3 \%-8 \%$ or $4 \%-12 \%$ gradient SDS-PAGE (NuPAGE; Invitrogen) and transferred onto an Immobilon-P (Millipore) membrane. Western blots were probed with antibodies to Atrx (1:10 dilution, F39; gift of D. Higgs, University of Oxford, Oxford, United Kingdom), $\alpha$-tubulin (1:10,000 dilution, clone DM1A; Sigma-Aldrich), p53 (1:2,000 dilution, 1C12; Cell Signaling), p53 (ab6) (1:1,000 dilution; Calbiochem), phospho-p53 $3^{\text {Ser37 }}$ (1:500 dilution, 9289; Cell Signaling), $\beta$-actin (1:10,000 dilution, AC-15; Sigma-Aldrich), phospho-ATM ${ }^{\text {Ser1981 }}$ (10H11. E12; Millipore), Chk1 (1:1,000 dilution, 2G1D5; Cell Signaling), phosphoChk $1^{\text {Ser345 }}$ (2341; Cell Signaling), $\gamma$-H2AX (1:1,000 dilution, 20E3, Cell Signaling), Rad51 (1:1,000 dilution, R-1528; Sigma-Aldrich), PAR (1:2,000 dilution, 4335-MC-100; Trevigen), MHC (1:50 dilution, MF20; DSHB), cyclin E (1:3,000 dilution, 06459; Upstate), p107 (c18) (1:2,000 dilution; Santa Cruz), cyclin A (c19) (1:2,000 dilution; Santa Cruz), and Kip1/p27 (1:4,000 dilution, BD610241; BD Biosciences). Western blots were quantified by densitometry analysis using Image J software. See complete unedited blots in the supplemental material.

BrdU pulse-chase protocol. Primary myoblasts were infected with Ad-LacZ or Ad-Cre and the following day seeded at $4 \times 10^{5}$ cells per 10 -cm plate. Seeded myoblasts were maintained in GM for an additional 48 hours and then pulsed with $10 \mu \mathrm{M}$ BrdU for 1 hour. Myoblasts were washed 3 times with PBS, and fresh GM was reapplied. Myoblasts were trypsinized and fixed on ice-cold $70 \%$ ethanol at 4-hour intervals up to 24 hours. Fixed pulse-chased samples were processed for flow cytometry analysis as described previously (32). BrdU antibody (1:100 dilution, clone B44; BD Biosciences) and Alexa Fluor 488 secondary antibody (1:500 dilution; Invitrogen) were used. Fluorescence-activated flow cytometry was performed on a Beckman Coulter FACS Station, and cell cycle data were analyzed by ModFit software (Verity Software House). siRNA knockdown. Human primary myoblasts were seeded into $60-\mathrm{mm}$ tissue culture plates in myoblast GM. On the day of transfection, myoblasts were transfected in serum-free OptiMEM media (Invitrogen) with nontargeting siScrambled (siGENOME Non-Targeting siRNA Pool \#2; Dharmacon) or siATRX (siGENOME SMARTpool, Human ATRX; Dharmacon) at a concentration of $100 \mathrm{nM}$ with INTERFERin siRNA transfection reagent (Polyplus-transfection) according to the manufacturer's instructions.

Metaphase chromosome telomeric FISH. 48 hours after infection, primary myoblasts were treated with $10 \mu \mathrm{g} / \mathrm{ml}$ colchicine (Sigma-Aldrich) for 5 hours. Myoblasts were harvested and processed for telomeric PNA FISH (BioSynthesis) according to a previously published protocol (73).

SYBR QPCR analysis. RNA was harvested 48 hours after infection (Qiagen), and $1 \mu \mathrm{g}$ RNA was reverse transcribed (SuperScriptIII; Invitrogen). cDNA was used for expression analysis of cell cycle genes by qPCR SuperArray (PAMM020, SABiosciences). QPCR was performed on a Stratagene Mx3000P system.

WST-1 proliferation assay. Mouse or human myoblasts were seeded into a 96-well flat-bottomed cell culture plate (Costar) at $2 \times 10^{3}$ cells/well. Cells were maintained in GM for 4 days (mouse) and 5 days (human), at which time WST-1 assay (ab65473; Abcam) was performed according to the manufacturer's instructions.

Statistics. Statistical analyses were performed using Microsoft Excel. 2 -tailed $t$ test and $95 \%$ confidence intervals were used to determine significance of differences. A $P$ value less than 0.05 was considered significant.

Study approval. All mice used in our studies were housed and cared for according to Canadian Council on Animal Care (CCAC) guidelines and University of Ottawa Animal Care Committee protocols. Human lymphoblastoid cell lines were obtained after receipt of written informed consent from participants and guardians as outlined in the approved Ottawa Hospital Research Ethics Board Protocol no. 1998577-01H.

\section{Acknowledgments}

The authors thank Vanessa Seale for technical assistance with CTX muscle injections; Jeff Hamill for technical assistance with the flow cytometry; and M.A.M. Todd, F.J. Dilworth, and L. Megeney for comments on the manuscript. This work was supported by operating grants to D.J. Picketts from Canadian Institutes of Health Research and Association Francaise Contre les Myopathies.

Received for publication March 7, 2012, and accepted in revised form September 6, 2012.

Address correspondence to: David J. Picketts, Ottawa Hospital Research Institute, Regenerative Medicine Program, 501 Smyth Road, Ottawa, Ontario K1H8L6, Canada. Phone: 613.737.8989; Fax: 613.737.8803; E-mail: dpicketts@ohri.ca.
1. Gibbons R. Alpha thalassaemia-mental retardation, X linked. Orphanet J Rare Dis. 2006;1:15.

2. Medina CF, et al. Altered visual function and interneuron survival in Atrx knockout mice: inference for the human syndrome. Hum Mol Genet. 2009;18(5):966-977.

3. Bérubé NG, et al. The chromatin-remodeling protein ATRX is critical for neuronal survival during corticogenesis. J Clin Invest. 2005;115(2):258-267.
4. Bagheri-Fam S, et al. Defective survival of proliferating Sertoli cells and androgen receptor function in a mouse model of the ATR-X syndrome. Hum Mol Genet. 2011;20(11):2213-2224.

5. Baumann C, Viveiros MM, De La Fuente R. Loss of maternal ATRX results in centromere instability and aneuploidy in the mammalian oocyte and pre-implantation embryo. PLoS Genet. 2010;6(9):e1001137.

6. Picketts DJ, Tastan AO, Higgs DR, Gibbons RJ.
Comparison of the human and murine ATRX gene identifies highly conserved, functionally important domains. Mamm Genome. 1998;9(5):400-403.

7. Dhayalan A, et al. The ATRX-ADD domain binds to $\mathrm{H} 3$ tail peptides and reads the combined methylation state of K4 and K9. Hum Mol Genet. 2011; 20(11):2195-2203.

8. Iwase S, et al. ATRX ADD domain links an atypical histone methylation recognition mechanism 
to human mental-retardation syndrome. Nat Struct Mol Biol. 2011;18(7):769-776

9. Eustermann S, et al. Combinatorial readout of histone $\mathrm{H} 3$ modifications specifies localization of ATRX to heterochromatin. Nat Struct Mol Biol. 2011; 18(7):777-782.

10. Xue Y, et al. The ATRX syndrome protein forms a chromatin-remodeling complex with Daxx and localizes in promyelocytic leukemia nuclear bodies. Proc Natl Acad Sci US A. 2003;100(19):10635-10640.

11. Tang J, et al. A novel transcription regulatory complex containing death domain-associated protein and the ATR-X syndrome protein. J Biol Chem. 2004;279(19):20369-20377.

12. Piatti P, Zeilner A, Lusser A. ATP-dependent chromatin remodeling factors and their roles in affecting nucleosome fiber composition. Int J Mol Sci. 2011;12(10):6544-6565.

13. Talbert PB, Henikoff S. Histone variants--ancient wrap artists of the epigenome. Nat Rev Mol Cell Biol. 2010;11(4):264-275

14. Costanzi C, Pehrson JR. Histone macroH2A1 is concentrated in the inactive $\mathrm{X}$ chromosome of female mammals. Nature. 1998;393(6685):599-601.

15. Mito Y, Henikoff JG, Henikoff S. Genome-scale profiling of histone $\mathrm{H} 3.3$ replacement patterns. Nat Genet. 2005;37(10):1090-1097.

16. Jin $\mathrm{C}$, et al. H3.3/H2A.Z double variant-containing nucleosomes mark "nucleosome-free regions" of active promoters and other regulatory regions. Nat Genet. 2009;41(8):941-945.

17. Goldberg AD, et al. Distinct factors control histone variant $\mathrm{H} 3.3$ localization at specific genomic regions. Cell. 2010;140(5):678-691.

18. Drané P, Ouararhni K, Depaux A, Shuaib M, Hamiche A. The death-associated protein DAXX is a novel histone chaperone involved in the replication-independent deposition of H3.3. Genes Dev. 2010;24(12):1253-1265

19. Wong LH, et al. ATRX interacts with H3.3 in maintaining telomere structural integrity in pluripotent embryonic stem cells. Genome Res. 2010; 20(3):351-360.

20. Law MJ, et al. ATR-X syndrome protein targets tandem repeats and influences allele-specific expression in a size-dependent manner. Cell. 2010; 143(3):367-378.

21. Steensma DP, Higgs DR, Fisher CA, Gibbons RJ. Acquired somatic ATRX mutations in myelodysplastic syndrome associated with alpha thalassemia (ATMDS) convey a more severe hematologic phenotype than germline ATRX mutations. Blood. 2004;103(6):2019-2026.

22. Nelson ME, Thurmes PJ, Hoyer JD, Steensma DP. A novel 5' ATRX mutation with splicing consequences in acquired alpha thalassemia-myelodysplastic syndrome. Haematologica. 2005;90(11):1463-1470.

23. Jiao Y, et al. DAXX/ATRX, MEN1, and mTOR pathway genes are frequently altered in pancreatic neuroendocrine tumors. Science. 2011; 331(6021):1199-1203.

24. Heaphy CM, et al. Altered telomeres in tumors with ATRX and DAXX mutations. Science. 2011; 333(6041):425.

25. Gibbons RJ, Higgs DR. Molecular-clinical spectrum of the ATR-X syndrome. Am J Med Genet. 2000; 97(3):204-212

26. Tallquist MD, Weismann KE, Hellström M, Soriano P. Early myotome specification regulates PDGFA expression and axial skeleton development. Development. 2000;127(23):5059-5070.

27. Garrick D, et al. Loss of Atrx affects trophoblast development and the pattern of $\mathrm{X}$-inactivation in extraembryonic tissues. PLoS Genet. 2006;2(4):e58.

28. Kuang S, Gillespie MA, Rudnicki MA. Niche regulation of muscle satellite cell self-renewal and differentiation. Cell Stem Cell. 2008;2(1):22-31.
29. Gayraud-Morel B, et al. A role for the myogenic determination gene Myf5 in adult regenerative myogenesis. Dev Biol. 2007;312(1):13-28.

30. Kuang S, Kuroda K, Le Grand F, Rudnicki MA Asymmetric self-renewal and commitment of satellite stem cells in muscle. Cell. 2007;129(5):999-1010.

31. Ritchie $\mathrm{K}$, et al. Loss of ATRX leads to chromosome cohesion and congression defects. J Cell Biol. 2008;180(2):315-324.

32. Stubbert LJ, et al. DDB2-independent role for p53 in the recovery from ultraviolet light-induced replication arrest. Cell Cycle. 2007;6(14):1730-1740.

33. Ishov AM, Vladimirova OV, Maul GG. Heterochromatin and ND10 are cell-cycle regulated and phosphorylation-dependent alternate nuclear sites of the transcription repressor Daxx and SWI/SNF protein ATRX. J Cell Sci. 2004;117(pt 17):3807-3820.

34. Jackson SP, Bartek J. The DNA-damage response in human biology and disease. Nature. 2009; 461(7267): 1071-1078

35. Foijer F, Draviam VM, Sorger PK. Studying chromosome instability in the mouse. Biochim Biophys Acta. 2008;1786(1):73-82.

36. Cheung ALM, Deng W. Telomere dysfunction, genome instability and cancer. Front Biosci. 2008; 13:2075-2090.

37. Lovejoy CA, Cortez D. Common mechanisms of PIKK regulation. DNA Repair (Amst). 2009;8(9):1004-1008.

38. Serrano MA, et al. DNA-PK, ATM and ATR collaboratively regulate $\mathrm{p} 53-\mathrm{RPA}$ interaction to facilitate homologous recombination DNA repair [published online ahead of print July 16, 2012]. Oncogene. doi:10.1038/onc.2012.257.

39. Megeney LA, Kablar B, Garrett K, Anderson JE, Rudnicki MA. MyoD is required for myogenic stem cell function in adult skeletal muscle. Genes Dev. 1996;10(10):1173-1183

40. Solomon LA, Li JR, Bérubé NG, Beier F. Loss of ATRX in chondrocytes has minimal effects on skeletal development. PLoS ONE. 2009;4(9):e7106.

41. Kassar-Duchossoy L, et al. Pax3/Pax7 mark a novel population of primitive myogenic cells during development. Genes Dev. 2005;19(12):1426-1431.

42. Rudnicki MA, et al. MyoD or Myf-5 is required for the formation of skeletal muscle. Cell. 1993; 75(7):1351-1359

43. Kassar-Duchossoy L, et al. Mrf4 determines skeletal muscle identity in Myf5:Myod double-mutant mice. Nature. 2004;431(7007):466-471.

44. Relaix F, Rocancourt D, Mansouri A, Buckingham M. A Pax3/Pax7-dependent population of skeletal muscle progenitor cells. Nature. 2005;435(7044):948-953.

45. Gros J, Manceau M, Thomé V, Marcelle C. A common somitic origin for embryonic muscle progenitors and satellite cells. Nature. 2005;435(7044):954-958.

46. Gokhin DS, Ward SR, Bremner SN, Lieber RL. Quantitative analysis of neonatal skeletal muscle functional improvement in the mouse. J Exp Biol. 2008;211(pt 6):837-843

47. White RB, Biérinx A-S, Gnocchi VF, Zammit PS. Dynamics of muscle fibre growth during postnatal mouse development. BMC Dev Biol. 2010;10:21.

48. Parise G, McKinnell IW, Rudnicki MA. Muscle satellite cell and atypical myogenic progenitor response following exercise. Muscle Nerve. 2008 37(5):611-619.

49. Harris JB. Myotoxic phospholipases A2 and the regeneration of skeletal muscles. Toxicon. 2003; 42(8):933-945

50. Joe AWB, et al. Muscle injury activates resident fibro/adipogenic progenitors that facilitate myogenesis. Nat Cell Biol. 2010;12(2):153-163.

51. Frescas D, et al. KDM2A represses transcription of centromeric satellite repeats and maintains the heterochromatic state. Cell Cycle. 2008;7(22):3539-3547.

52. Michishita E, et al. SIRT6 is a histone H3 lysine 9 deacetylase that modulates telomeric chromatin.
Nature. 2008;452(7186):492-496

53. Jones B, et al. The histone H3K79 methyltransferase Dot $1 \mathrm{~L}$ is essential for mammalian development and heterochromatin structure. PLoS Genet. 2008;4(9):e1000190.

54. Palacios JA, et al. SIRT1 contributes to telomere maintenance and augments global homologous recombination. J Cell Biol. 2010;191(7):1299-1313.

55. Collins N, et al. An ACF1-ISWI chromatinremodeling complex is required for DNA replication through heterochromatin. Nat Genet. 2002; 32(4):627-632.

56. Poot RA, et al. The Williams syndrome transcription factor interacts with PCNA to target chromatin remodelling by ISWI to replication foci. Nat Cell Biol. 2004;6(12):1236-1244.

57. Rowbotham SP, et al. Maintenance of silent chromatin through replication requires SWI/SNF-like chromatin remodeler SMARCAD1. Mol Cell. 2011; 42(3):285-296.

58. Sims JK, Wade PA. Mi-2/NuRD complex function is required for normal $\mathrm{S}$ phase progression and assembly of pericentric heterochromatin. Mol Biol Cell. 2011;22(17):3094-3102.

59. Lewis PW, Elsaesser SJ, Noh K-M, Stadler SC, Allis CD. Daxx is an H3.3-specific histone chaperone and cooperates with ATRX in replication-independent chromatin assembly at telomeres. Proc Natl Acad Sci U S A. 2010;107(32):14075-14080.

60. Sampathi S, Chai W. Telomere replication: poised but puzzling. J Cell Mol Med. 2011;15(1):3-13

61. Wu Y, Brosh RM Jr. G-quadruplex nucleic acids and human disease. FEBS J. 2010;277(17):3470-3488.

62. Rizzo A, et al. Stabilization of quadruplex DNA perturbs telomere replication leading to the activation of an ATR-dependent ATM signaling pathway. Nucleic Acids Res. 2009;37(16):5353-5364.

63. Maher RL, Branagan AM, Morrical SW. Coordination of DNA replication and recombination activities in the maintenance of genome stability. $J$ Cell Biochem. 2011;112(10):2672-2682.

64. Alexiadis V, Kadonaga JT. Strand pairing by Rad54 and Rad51 is enhanced by chromatin. Genes Dev. 2002;16(21):2767-2771.

65. Jaskelioff M, Van Komen S, Krebs JE, Sung P, Peterson CL. Rad54p is a chromatin remodeling enzyme required for heteroduplex DNA joint formation with chromatin. J Biol Chem. 2003;278(11):9212-9218.

66. Tan TL, et al. Mouse Rad54 affects DNA conformation and DNA-damage-induced Rad51 foci formation. Curr Biol. 1999;9(6):325-328.

67. Golub EI, Kovalenko OV, Gupta RC, Ward DC, Radding CM. Interaction of human recombination proteins Rad51 and Rad54. Nucleic Acids Res. 1997;25(20):4106-4110.

68. Verdun RE, Crabbe L, Haggblom C, Karlseder J. Functional human telomeres are recognized as DNA damage in G2 of the cell cycle. Mol Cell. 2005; 20(4):551-561.

69. Verdun RE, Karlseder J. The DNA damage machinery and homologous recombination pathway act consecutively to protect human telomeres. Cell. 2006;127(4):709-720.

70. Kuang S, Chargé SB, Seale P, Huh M, Rudnicki MA. Distinct roles for Pax7 and Pax3 in adult regenerative myogenesis. J Cell Biol. 2006;172(1):103-113.

71. Huh MS, Parker MH, Scimè A, Parks R, Rudnicki $\mathrm{MA} . \mathrm{Rb}$ is required for progression through myogenic differentiation but not maintenance of terminal differentiation. J Cell Biol. 2004;166(6):865-876.

72. Rosenblatt JD, Lunt AI, Parry DJ, Partridge TA. Culturing satellite cells from living single muscle fiber explants. In Vitro Cell Dev Biol Anim. 1995; 31(10):773-779.

73. Lansdorp PM, et al. Heterogeneity in telomere length of human chromosomes. Hum Mol Genet. 1996;5(5):685-691. 